\title{
Endoscopic band ligation and over-the-scope clip placement for refractory bleeding of Dieulafoy ulcer in the jejunum
}

A 68-year-old man, who underwent surgery for gastric cancer (distal gastrectomy and Billroth I reconstruction) in the past and was taking dabigatran $15 \mathrm{mg}$ orally because of brain infarction, came to our hospital with a chief complaint of lightheadedness. He had marked anemia (hemoglobin $5.6 \mathrm{~g} / \mathrm{dL}$ ). Endoscopy detected bleeding from a Dieulafoy ulcer in the upper jejunum. A clip was applied to stop the bleeding ( $\mathbf{F i g . 1} \mathbf{~ a , b}$ ).

Thereafter, rebleeding occurred three times despite clip placement for hemostasis (- Fig. 1 c).

As the patient repeatedly bled, he was hospitalized to receive elective hemostasis by an over-the-scope clip (OTSC; Ovesco Endoscopy AG, Tübingen, Germany) 2 months after the 4th clipping. Endoscopy found that the clip had fallen off the lesion, which was oozing blood. We applied the OTSC. However, bleeding continued. We then performed endoscopic band ligation (EBL) right above the OTSC, and hemostasis was finally achieved ( $\triangleright$ Fig. 2 , - Video 1). Upper gastrointestinal endoscopy performed 1 month later confirmed scarring of the lesion ( $\triangleright$ Fig. $\mathbf{3}$ ). Clipping using an OTSC is effective as first-line and second-line treatment for peptic ulcers $[1,2]$. The hemostasis rate with OTSC is reported to be $64.7 \%$ $100 \%$ [3]. In some cases, EBL will incorporate even the muscularis propria and serosa of a lesion, with a high risk of perforation reported for lesions in the small intestine $[4,5]$. In the present case, we performed EBL right above the OTSC. We ligated the blood vessel safely by this method because the OTSC prevented EBL from including deep muscular layers of the lesion. In the future, this method may become an effective approach for gastrointestinal bleeding that is difficult to stop. To our knowledge, this is the first report of hemostasis using $\mathrm{EBL}$ in addition to an OTSC, and may be a valuable method of treating refractory gastrointestinal bleeding.

Endoscopy_UCTN_Code_TTT_1AO_2AD

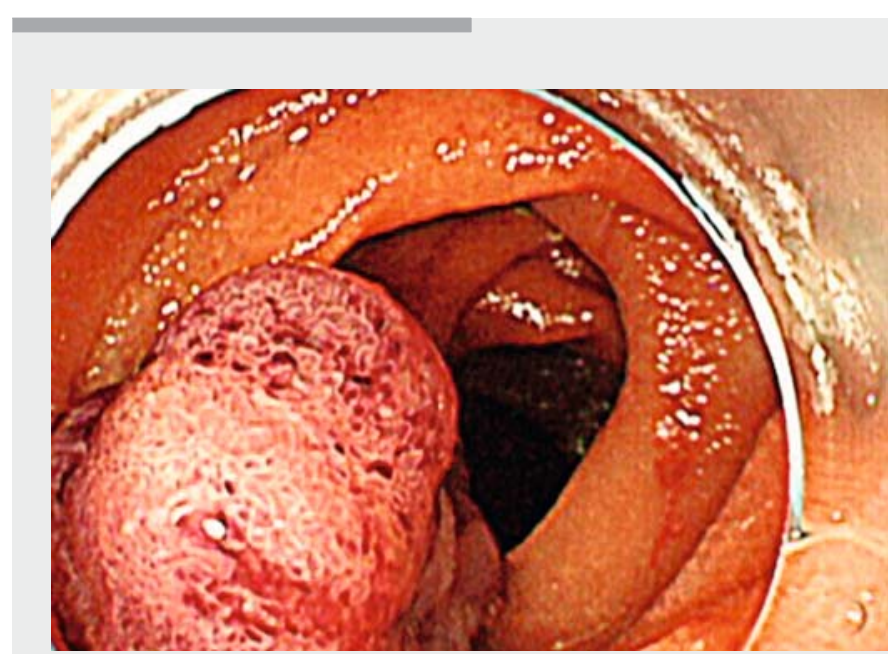

Video 1 A hemostatic procedure using an over-the-scope clip (OTSC; Ovesco Endoscopy AG, Tübingen, Germany) and endoscopic band ligation for refractory bleeding of a Dieulafoy ulcer in the jejunum.
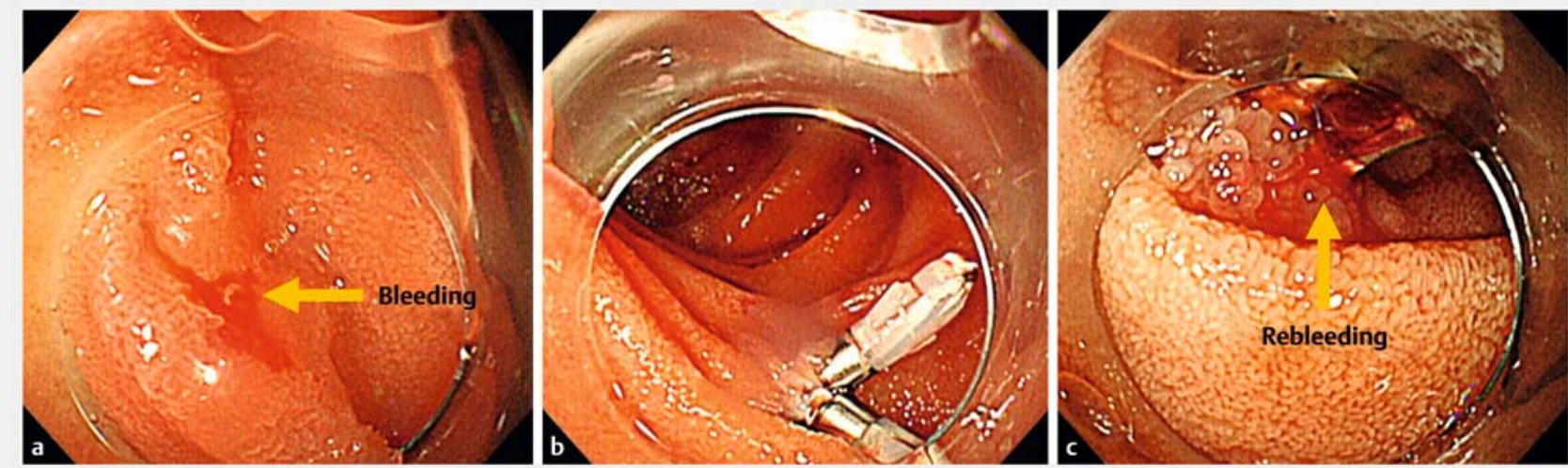

- Fig. 1 Endoscopic images. a Bleeding was detected from a Dieulafoy ulcer in the jejunum. b A clip was applied to stop the bleeding. c Rebleeding occurred despite clip placement. 


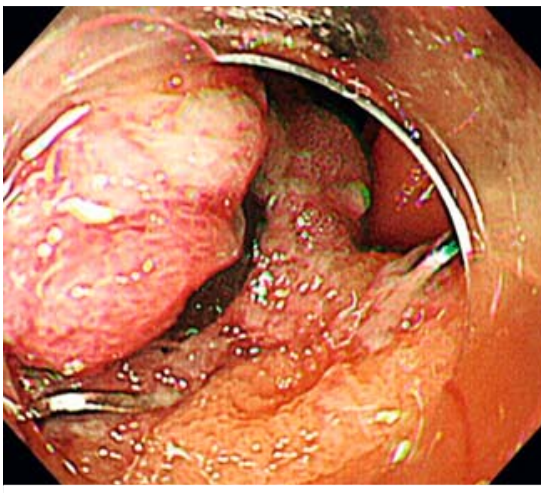

- Fig. 2 Endoscopic band ligation was performed right above the over-the-scope clip (OTSC; Ovesco Endoscopy AG, Tübingen, Germany) and hemostasis was finally achieved.

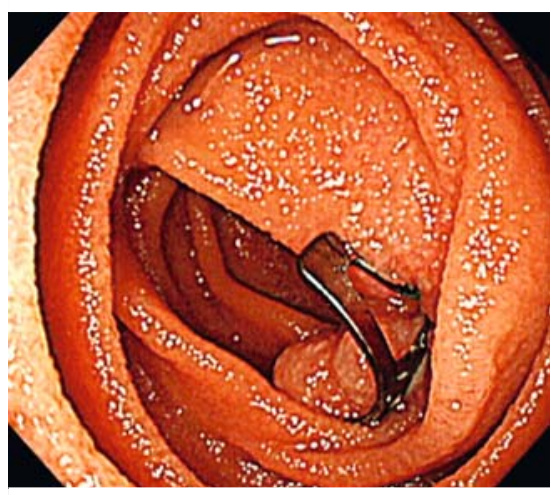

- Fig. 3 Scarring of Dieulafoy ulcer in the jejunum was confirmed.

\section{Competing interests}

The authors declare that they have no conflict of interest.

\section{The authors}

Takafumi Yano ${ }^{1}$, Takayuki Nagai ${ }^{1}$, Koki Yamanaka', Masato Hanzawa', Kosuke Kodama ${ }^{1}$, Takashi Abe', Kazunari Murakami ${ }^{2}$

1 Department of Gastroenterology, Oita Kouseiren Tsurumi Hospital, Beppu, Japan

2 Department of Gastroenterology, Faculty of Medicine, Oita University, Yufu, Japan

\section{Corresponding author}

\section{Takafumi Yano, MD, PhD}

Department of Gastroenterology, Oita Kouseiren Tsurumi Hospital, Tsurumi 4333 , Beppu City, Oita 874-8585, Japan Fax: +81-977-237884 yano1371@kitasato-u.ac.jp

\section{References}

[1] Manta R, Mangiafico S, Zullo A et al. Firstline endoscopic treatment with over-thescope clips in patients with either upper or lower gastrointestinal bleeding: a multicenter study. Endosc Int Open 2018; 6: E1317E1321

[2] Stefan G, Lukas N, Denis F et al. Over-thescope clip in peptic ulcer bleeding: clinical success in primary and secondary treatment and factors associated with treatment failure. Endosc Int Open 2019; 7: E846-E854

[3] Zhong C, Tan S, Ren Y et al. Clinical outcomes of over-the-scope-clip system for the treatment of acute upper non-variceal gastrointestinal bleeding: a systematic review and meta-analysis. BMC Gastroenterol 2019; 19: 225

[4] Barker KB, Arnold HL, Fillman EP et al. Safety of band ligator use in the small bowel and the colon. Gastrointest Endosc 2005; 62: 224-227

[5] Kakutani H, Sasaki S, Ueda K et al. Is it safe to perform endoscopic band ligation for the duodenum? A pilot study in ex vivo porcine models. Minim Invasive Ther Allied Technol 2013; 22: 80-83

Bibliography

Endoscopy 2021; 53: E85-E86

DOI 10.1055/a-1195-1795

ISSN 0013-726X

published online 26.6 .2020

(c) 2020. Thieme. All rights reserved.

Georg Thieme Verlag KG, Rüdigerstraße 14, 70469 Stuttgart, Germany

\section{ENDOSCOPY E-VIDEOS}

https://eref.thieme.de/e-videos

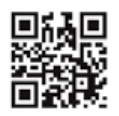

Endoscopy E-Videos is a free access online section, reporting on interesting cases and new techniques in gastroenterological endoscopy. All papers include a high quality video and all contributions are freely accessible online.

This section has its own submission website at https://mc.manuscriptcentral.com/e-videos 